\title{
EXTENDING THE UTILITY OF PERTURBATION SERIES IN PROBLEMS OF LAMINAR FLOW IN A POROUS PIPE AND A DIVERGING CHANNEL
}

\author{
O. D. MAKINDE ${ }^{1}$ \\ (Received 13 May 1996; revised 3 March 1998)
}

\begin{abstract}
In this paper, we exploit a new series summation and convergence improvement technique (that is, Drazin and Tourigny [5]), in order to study the steady flow of a viscous incompressible fluid both in a porous pipe with moving walls and an exponentially diverging asymmetrical channel. The solutions are expanded into Taylor series with respect to the corresponding Reynolds number. Using the D-T method, the bifurcation and the internal flow separation studies are performed.
\end{abstract}

\section{Introduction}

For over four decades, workers in statistical mechanics have effectively employed the technique of extending a regular perturbation series to high order by computer, and then analysing the coefficients to reveal the structure of the solution (Gaunt and Gutmann [8]). That procedure was later adapted to a variety of problems in fluid mechanics (Van Dyke [6]). Recently, Drazin and Tourigny [5] presented a novel computational approach to the investigation of bifurcations that relies on the use of power series in the bifurcation parameter for a particular solution branch. Their initial motivation was to solve boundary-value problems for non-linear systems of ordinary and partial differential equations. The procedure leads to a special type of Hermite-Padé approximant. Let us suppose that the partial sum

$$
U_{N}(\lambda)=\sum_{n=1}^{N} a_{n} \lambda^{n}=U(\lambda)+O\left(\lambda^{N+1}\right) \quad \text { as } \lambda \rightarrow 0
$$

\footnotetext{
${ }^{1}$ Applied Mathematics Department, University of the North, Private Bag X1106, Sovenga 0727, South Africa

(C) Australian Mathematical Society 1999, Serial-fee code 0334-2700/99
} 
is given. We shall make the simplest hypothesis in the context of non-linear problems by assuming that $U(\lambda)$ is the local representation of an algebraic function $u$ of $\lambda$. Therefore, we seek a polynomial $F_{d}=F_{d}(\lambda, u)$ of degree $d \geq 2$,

$$
F_{d}(\lambda, u)=\sum_{m=1}^{d} \sum_{k=0}^{m} f_{m-k, k} \lambda^{m-k} u^{k},
$$

such that

$$
\frac{\partial F_{d}}{\partial u}(0,0)=1
$$

and

$$
F_{d}\left(\lambda, U_{N}(\lambda)\right)=O\left(\lambda^{N+1}\right) \quad \text { as } \lambda \rightarrow 0 .
$$

Condition (1.3), which yields $f_{0,1}=1$, ensures that the polynomial $F_{d}$ has only one root which vanishes at $\lambda=0$ and also normalises $F_{d}$. There are thus

$$
1+\sum_{m=2}^{d}(m+1)=\frac{1}{2}\left(d^{2}+3 d-2\right)
$$

undetermined coefficients in the polynomial (1.2). The requirement (1.4) reduces the problem to a system of $N$ linear equations for the unknown coefficients of $F_{d}$. The entries of the underlying matrix depend only on the $N$ given coefficients $a_{n}$. Henceforth, we shall take

$$
N=\frac{1}{2}\left(d^{2}+3 d-2\right)
$$

so that the number of equations equals the number of unknowns. A bifurcation occurs where the solutions of a non-linear system change their qualitative character as a parameter changes. In particular, bifurcation theory is about how the number of steady solutions of a system depends on a parameter. The bifurcations for $F_{d}(\lambda, u)$ can then be analysed locally by means of Newton's diagram (Vainberg and Trenogin [18]).

The objective in the present paper is to demonstrate the applicability of the D-T method in solving non-linear systems of ordinary and partial differential equations. In Section 2, we consider the steady flow in a porous pipe with axially decelerating walls (Berman [1]). The problem reveals the procedure's ability to find disconnected solution branches, and also serves to illustrate how strongly non-linear results can be found accurately by using only weakly non-linear results. In Sections 3, we investigate the problem of steady flow in an exponentially diverging asymmetrical channel (Blasius [2]). The problem is considered in the boundary-layer approximation and reveals the suitability of Padé approximants over the D-T method in determining the position of internal flow separation. In Section 4, we discuss the pertinent results. 


\section{Porous pipe with decelerating walls flow}

The steady axisymmetric flow of a viscous incompressible fluid driven along a pipe by the combined effect of the wall deceleration and suction is considered. This type of problem was first investigated by Berman [1] and subsequently by many authors, for example, Terrill and Thomas [17], Zaturska and Banks [19], Makinde [11]. Let $E$ be a parameter such that the axial velocity of the wall is $E z$. It is assumed that $a E / V=O(1)$ and $V \neq 0(V>0$ represents suction velocity and $V<0$ represents injection velocity).

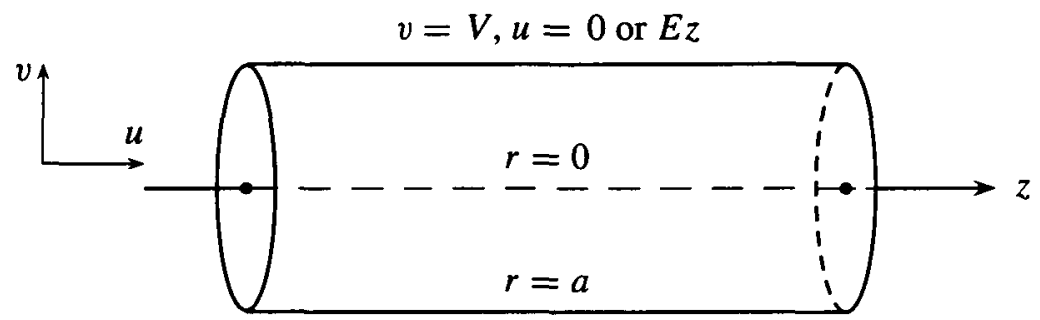

FIGURE 1. Schematic diagram of the problem.

By assuming a similarity form for the solution of the Navier-Stokes equations it is found, after non-dimensionalization, that the velocity components $(u, v)$ increasing in the directions of $(z, r)$, respectively, and vorticity $\omega$ of the flow may be expressed as (Makinde [13])

$$
u=\frac{z}{r} \frac{d F}{d r}, \quad v=-\frac{1}{r} F, \quad \omega=-z G,
$$

and

$$
\begin{aligned}
& \frac{d}{d r}\left[\frac{1}{r} \frac{d}{d r}(r G)\right]=R\left[\frac{G}{r} \frac{d F}{d r}-F \frac{d}{d r}\left(\frac{G}{r}\right)\right], \quad G=\frac{d}{d r}\left(\frac{1}{r} \frac{d F}{d r}\right), \\
& F=0, \quad \frac{d}{d r}\left(\frac{1}{r} \frac{d F}{d r}\right)=0, r \quad \text { on } \quad r=0, \\
& F=-1, \quad \frac{d F}{d r}=-1, \quad \text { on } \quad r=1 .
\end{aligned}
$$

The Reynolds number associated with the flow is $R=E a^{2} / \nu$, where $a$ is the tube characteristic radius and $v$ is the kinematic viscosity of the fluid. The solutions $F=F(r, R)$ and $G=G(r, R)$ of the resulting boundary-value problem can be 
obtained in the form of power series in $R$, (that is, for small $R$ ),

$$
\begin{aligned}
& {\left[\frac{1}{r}\left(r G_{n}\right)^{\prime}\right]^{\prime}=R \sum_{i=0}^{n-1}\left[G_{i}\left(\frac{F_{n-i-1}^{\prime}}{r}\right)-F_{i}\left(\frac{G_{n-i-1}}{r}\right)^{\prime}\right], \quad G_{n}=\left(\frac{1}{r} F_{n}^{\prime}\right)^{\prime},} \\
& F_{n}=0, \quad\left(\frac{1}{r} F_{n}^{\prime}\right)^{\prime}=0, \quad \text { on } r=0, \\
& F_{0}=-1, F_{n+1}=0, F_{0}^{\prime}=-1, F_{n+1}^{\prime}=0, \quad \text { on } r=1, n=0,1,2, \ldots,
\end{aligned}
$$

where the prime symbol denotes differentiation with respect to $r$. We expand $F(r, R)$, $\beta=\left(F^{\prime} / r\right)^{\prime}$ at $r=1$ and $F^{\prime \prime}(0)$ (that is, stream-function, skin friction and centreline axial velocity parameter) in powers of the Reynolds number, to obtain

$$
\begin{aligned}
F(r) & =-\frac{1}{2} r^{2}\left(3-r^{2}\right)-\frac{1}{144} r^{2}\left(7-r^{2}\right)\left(1-r^{2}\right)^{2} R+\cdots, \\
\beta & =4-\frac{1}{3} R-\frac{2}{27} R^{2}-\frac{17}{1008} R^{3}-\cdots,
\end{aligned}
$$

and

$$
F^{\prime \prime}(0)=-3-\frac{7}{72} R-\frac{103}{4800} R^{2}-\frac{760589}{152409600} R^{3}-\cdots \quad \text { as } R \rightarrow 0 .
$$

Using a symbolic algebra package such as Maple, the first 54 coefficients of the solution series were obtained. We observed that the signs of the coefficients are the same and are monotonically decreasing in magnitude. The convergence of the series may be limited by a singularity on the positive real axis (Van Dyke [6]). The graphical form of the D'Alembert's ratio test (Domb and Sykes [4]) together with Neville's extrapolation at $1 / n=0$ (that is, $n \rightarrow \infty$ ) reveal the radius of convergence $R=3.07249$. Following the Drazin and Tourigny technique, we compute the first and second turning points $R_{1}$ and $R_{2}$ together with $\beta_{-1}$ and $F_{-1}^{\prime \prime}(0)$ on the secondary branch as $R \rightarrow 0$, since $\beta \sim \beta_{-1} R^{-1}$ and $F^{\prime \prime}(0) \sim F_{-1}^{\prime \prime}(0) R^{-1}$ as $R \rightarrow 0$ on the secondary branch (Brady and Acrivos [3]). Our results show that $R_{1} \approx 3.0724980042, R_{1} \approx 8.813114939$, $\beta \rightarrow-67.670206 R^{-1}$, and $F^{\prime \prime}(0) \rightarrow-35.3149734 R^{-1}$ as $R \rightarrow 0$ on the secondary branch (see Table 1). It is interesting to notice the absence of real solutions for $R_{1}<R<R_{2}$, and that $\beta \rightarrow 0$ as $R \rightarrow 2.828847 \ldots$, that is, reversal of the flow at the wall will occur (see Figure 3).

\section{Flow in a diverging channel}

The two-dimensional flow of a viscous incompressible fluid driven steadily through an exponentially diverging asymmetrical channel is considered. This problem was 
introduced into the literature by Blasius [2] and has been treated mathematically in several ways by many researchers, for example Fraenkel [7], Lucas [9], Pedley [16], Makinde [12], Makinde and Lungu [15].

TABLE 1. Computations for the flow in porous tube with axially decelerating walls.

\begin{tabular}{|l|l|c|c|c|c|}
\hline $\mathrm{d}$ & $\mathrm{N}$ & $R_{1}^{(d)}$ & $R_{2}^{(d)}$ & $\beta_{-1}^{(d)}$ & $F_{-1}^{\prime(d)}(0)$ \\
\hline 2 & 4 & 2.9908989594384452426 & & & \\
\hline 3 & 8 & 3.0729182958247960750 & & & \\
\hline 4 & 13 & 3.0724931718813353943 & 8.826676298 & -80.829517 & -34.7954971 \\
\hline 5 & 19 & 3.0724980037912682482 & 8.813139499 & -67.329194 & -35.3066983 \\
\hline 6 & 26 & 3.0724980042504038099 & 8.813109211 & -67.666689 & -35.3151076 \\
\hline 7 & 34 & 3.0724980042458196719 & 8.813114950 & -67.670223 & -35.3149729 \\
\hline 8 & 43 & 3.0724980042458197011 & 8.813114939 & -67.670206 & -35.3149734 \\
\hline 9 & 53 & 3.0724980042458197011 & 8.813114939 & -67.670206 & -35.3149734 \\
\hline
\end{tabular}

Let $u$ and $v$ be the velocity components increasing in the directions of $x$ and $y$ respectively, and $b(x)$ be the variation in the channel's width.

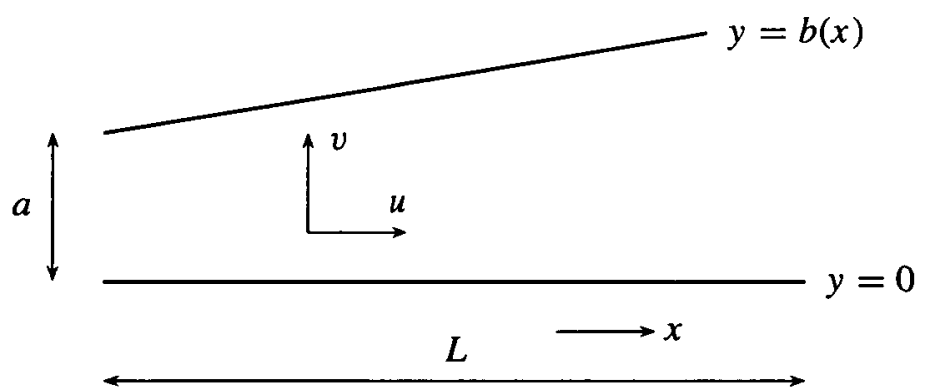

FIGURE 2. Schematic diagram of the problem.

The channel's width $(y=b(x)=a S(x / L))$ is assumed to vary slowly with axial distance such that $0<\varepsilon=a / L \ll 1$, where $a$ is the channel's characteristic width and $L$ is the characteristic length. In the limit $\varepsilon \rightarrow 0$, the channel is of constant width. The following dimensionless variables are introduced

$$
\bar{\omega}=\frac{a^{2} \omega}{Q}, \quad \bar{x}=\frac{\varepsilon x}{a}, \quad \bar{y}=\frac{y}{a}, \quad \bar{\Psi}=\frac{\Psi}{Q},
$$

where $Q$ is the constant flux across any cross-section of the channel, $\Psi$ is the streamfunction and $\omega$ is the vorticity. The flow is considered in the boundary layer approx- 
imation or for a channel with a small aspect ratio $\varepsilon$. The dimensionless governing equations together with the appropriate boundary conditions (neglecting the bars for clarity) can be written as (Makinde [14])

$$
\begin{aligned}
\frac{\partial^{2} \omega}{\partial y^{2}} & =\operatorname{Re} \frac{\partial(\omega, \Psi)}{\partial(x, y)}, \\
\omega & =-\frac{\partial^{2} \Psi}{\partial y^{2}}, \\
\Psi & =0, \quad \frac{\partial \Psi}{\partial y} \quad \text { on } \quad y=0, \\
\frac{\partial \Psi}{\partial y} & =0, \quad \Psi=1 \quad \text { on } \quad y=S,
\end{aligned}
$$

where $\operatorname{Re}=\varepsilon Q / \nu$ is the effective flow Reynolds number and we emphasize that $\operatorname{Re}=O(\varepsilon)$ as $\varepsilon \rightarrow 0$. For the geometry of the channel under consideration, $S$ is defined as $S=e^{x}$.

In order to estimate the position of separation in the flow field, the state variables $\Psi, \beta\left(=S^{2} \partial^{2} \Psi / \partial y^{2}\right)$, and $H\left(=S^{3} \varepsilon \partial P / \partial x, P\right.$ is the fluid pressure), that is, streamfunction, the wall shear stress and the axial pressure gradient parameters respectively, are expanded in powers of $h(=\operatorname{Re} S)$ to obtain

$$
\begin{gathered}
\Psi(x, y)=-\eta^{2}(2 \eta-3)-\frac{3}{35} \eta^{2}(2 \eta-1)\left(\eta^{2}-\eta-1\right)\left(\eta^{2}-1\right)^{2} h+\cdots, \\
\beta=6-\frac{6}{35} h-\frac{39}{26950} h^{2}+\frac{67}{6131135} h^{3}+\frac{575683}{914923259250} h^{4}+\cdots, \\
H=-12+\frac{54}{35} h+\frac{39}{13475} h^{2}-\frac{1044}{6131125} h^{3}-\frac{8564377}{1524872098750} h^{4}+\cdots
\end{gathered}
$$

as $h \rightarrow 0$ and where $\eta=y / s$. Using a computing aid such as Maple, the first 44 coefficients of the above series were obtained. We noticed that the coefficients of wall shear stress alternate regularly after the tenth term and are monotonically decreasing in magnitude (that is, similar to the Lucas [9] problem). We also recast the series into several diagonal Padé approximants $[M / M]$ (where $n=M+M$ is the order of the series required for each approximant). The D-T method is also utilised and a comparison of the results shows good agreement as the number of terms of the series used increases, as shown in the Table 2 below. Hence, the position of separation for any given Reynolds number Re is given by $S_{c} \approx 31.7397 / \mathrm{Re}$, that is, as $\beta \rightarrow 0$.

\section{Graphical results and discussion}

With the aid of Maple graphic facilities and using the D-T method, a sketch of the bifurcation diagram for the problem of a porous tube flow with decelerating wall was 
TABLE 2. Comparison of the Padé approximant with the D-T method in estimating the separation position for asymmetric flow in an exponentially diverging channel, that is, $h$ as $\beta \rightarrow 0$.

\begin{tabular}{|c|c|c|c|}
\hline$n$ & $m$ & $\begin{array}{c}\left(h_{c}=\operatorname{Re} S_{c}\right) \text { Padé } \\
\text { approximants }[M / M]\end{array}$ & $\begin{array}{c}\left(h_{c}=\operatorname{Re} S_{c}\right) \\
\text { D-T method }\end{array}$ \\
\hline 4 & 2 & & 29.99643400951 \\
\hline 8 & 4 & 31.75531685568 & 31.04359838975 \\
\hline 14 & 7 & 31.72949279707 & 31.89432463789 \\
\hline 20 & 10 & 31.74025328399 & 31.74548021256 \\
\hline 26 & 13 & 31.73969222172 & 31.73963919155 \\
\hline 34 & 17 & 31.73973143549 & 31.73973283481 \\
\hline 44 & 22 & 31.73971036233 & 31.73970885902 \\
\hline
\end{tabular}

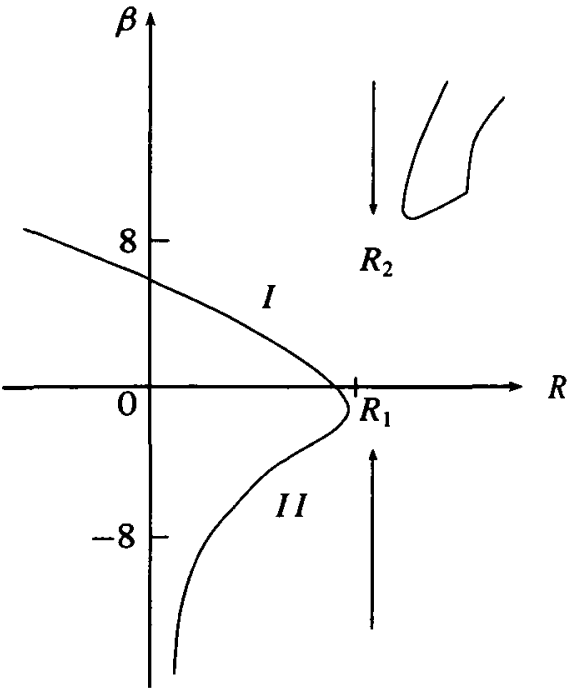

(a)

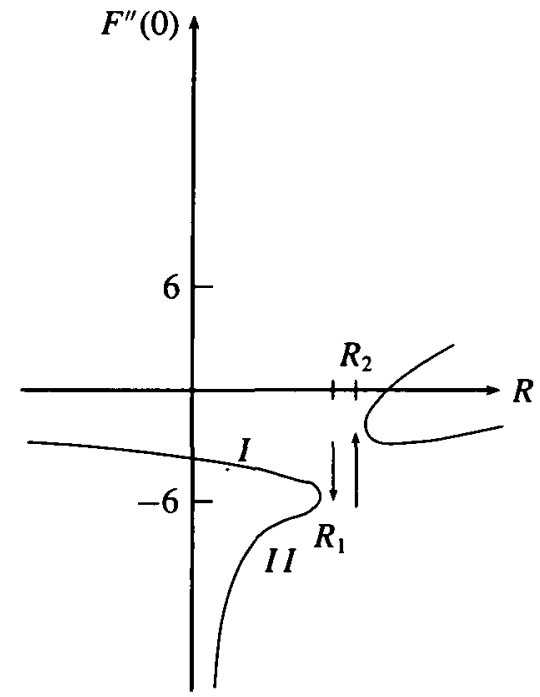

(b)

FIGURE 3. A sketch of the bifurcation diagram for the porous-tube-with-decelerating-wall flow problem; (a) skin friction, and (b) centreline axial velocity, with respect to $R$. 


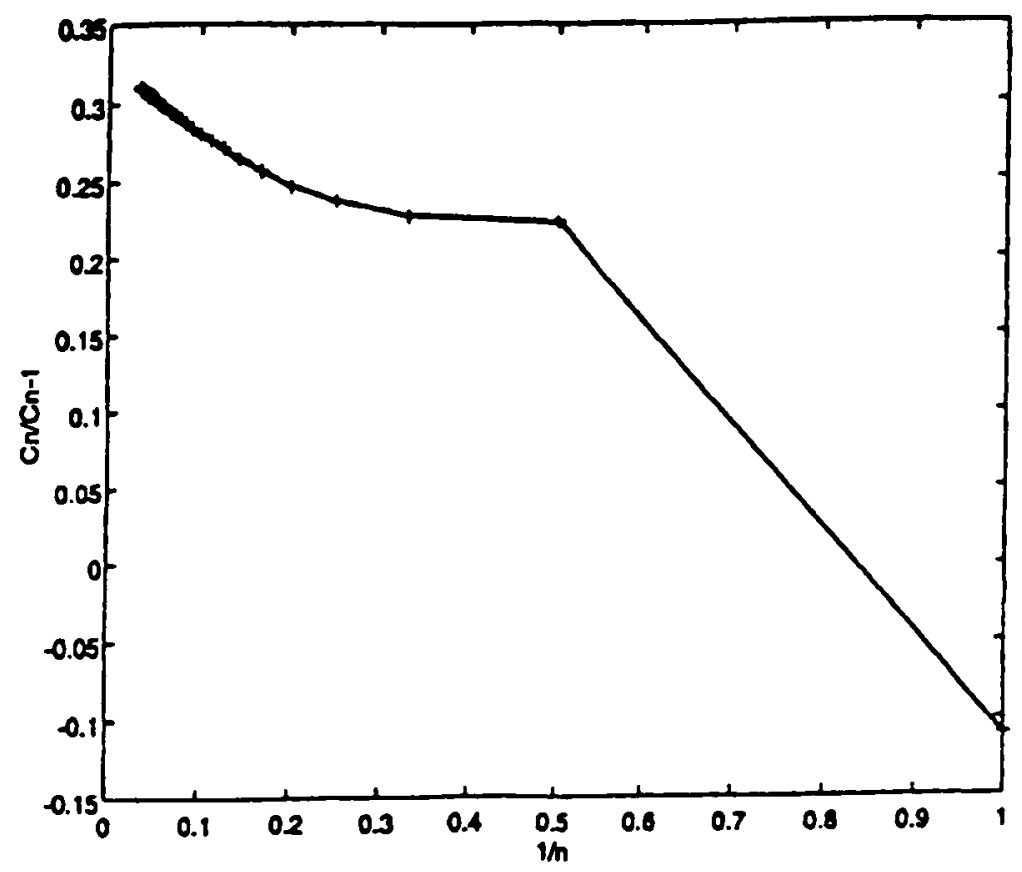

FIGURE 4. Domb-Sykes plot for coefficients of $\beta$ for the porous-tube flow problem (Radius of convergence $=3.07249$ ).

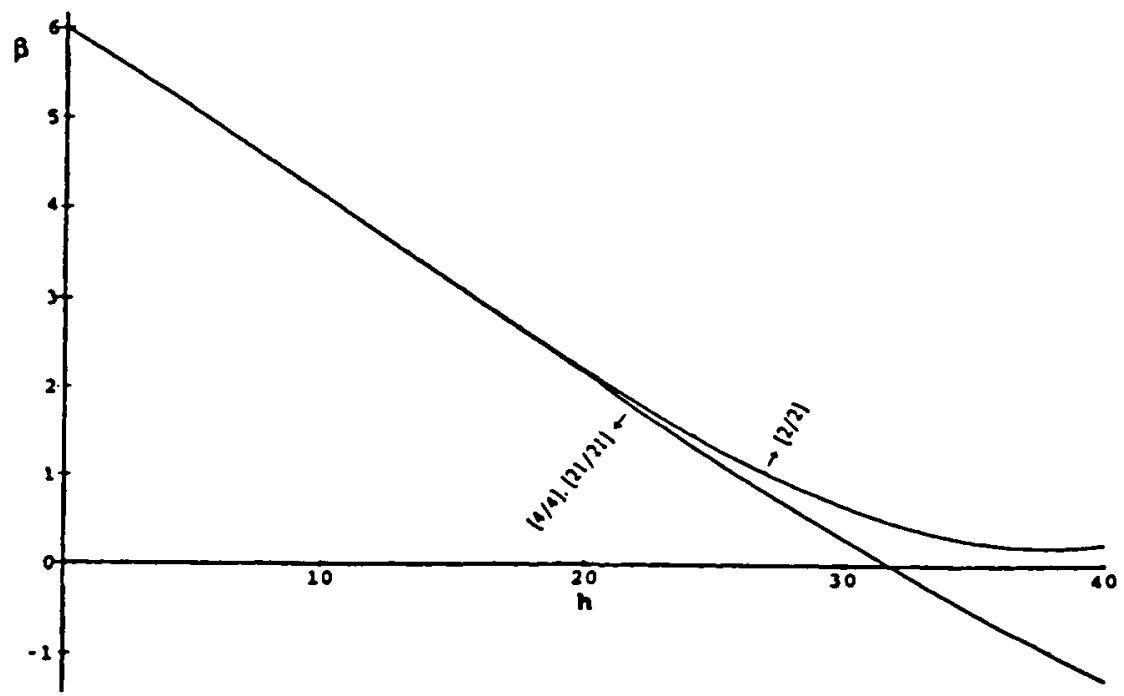

FIGURE 5. Variation of wall shear stress $(\beta)$ with respect to $h(=\operatorname{Re} S)$ for the exponentially-divergingasymmetrical-channel flow problem. 
obtained and is shown in Figure 4. We obtained accurately the two turning points $R_{1}, R_{2}$ as well as the asymptotic behaviour of skin friction and the centre-line axial velocity as $R \rightarrow 0$ on the secondary solution branch (see Table 1). The absence of real solutions for $R_{1}<R<R_{2}$ is noticed and consequently the branches with turning points $R_{1}$ and $R_{2}$ are disconnected. Figure 5 shows the Domb-Sykes plot for the coefficients of the skin friction $\beta$. The radius of convergence that corresponds to the nearest singularity lies on the positive real axis of $R$ and is given as $R=3.07249$.

Figure 6 shows the variation of the wall shear stress with respect to the parameter $h(=\operatorname{Re} S)$ using several diagonals Padé approximants, for steady flow in an exponentially asymmetrical channel. We observe a convergence from the [4/4] Padé approximant, and the wall shear stress decreases with an increase in $h$. The position of internal flow separation is given by $S_{c} \approx 31.7397 / \operatorname{Re}$ (see Table 2 ). Here, we observe that our result at the separation position is four times the result obtained by Lucas [9] at the separation position (that is, $4 \times 7.9349=31.739$ ). This implies that the results obtained for flow in an asymmetrical channel can be easily transformed to those in a symmetrical channel (Makinde [14]).

Finally, the porous tube flow problem investigated in Section 2 demonstrates the ability of the D-T method to find disconnected solution branches and also serves to illustrate how strongly non-linear results can be found accurately by using only weakly non-linear results. However, in the case of an exponentially diverging asymmetrical channel no bifurcation was found. Also we observe that the Padé approximants converge better than the D-T method in the absence of turning points, although the Padé approximants can only improve the result on the single solution branch representing the original power series. We emphasize that the D-T method is, in essence, a numerical form of analytic continuation and, as such, can only be expected to reveal those branches that are analytic continuations of the original power series. Furthermore, it is noteworthy that the applicability of the D-T method as well as other series summation and improvement techniques depend on the availability of partial sums of the solution series for the problem under consideration. In many situations, it is possible to obtain the Taylor coefficients exactly (for instance by perturbation methods). Reliance upon the exactness of the Taylor coefficients may, however, limit the usefulness of the procedure to a rather small portion of the global bifurcation diagram. Hence, one may compute the approximate Taylor coefficients by using the standard numerical part-following techniques (Lyness, [10]), thus greatly enhancing the scope and range of applicability of the D-T method. The D-T method has a wide range of application and may fail to reveal the bifurcation point for some problems (for instance when the pattern of signs of the coefficients of solution series is irregular or cannot be easily established), but whenever it works, the error decays faster than exponentially with the number of terms of the series used, as illustrated by our example. Therefore, it is not a panacea for all applied-mathematics problems. 
Moreover, the D-T method utilised in this paper is obviously amenable to various types of generalizations. For instance, it is quite straightforward to devise extensions to cater for problems with more than one bifurcation parameter or with more than one scalar state variable. Such generalizations may, however, be very demanding of computing time and memory.

\section{Acknowledgements}

The author thanks Prof. P. G. Drazin, School of Mathematics, University of Bristol, United Kingdom, for his encouragement during the preparation of this paper, the referees for their useful suggestions and the Commonwealth Scholarship Commission in the United Kingdom for the financial support.

\section{References}

[1] A. S. Berman, "Laminar flow in channels with porous walls", J. Appl. Phys. 24 (1953) 1232-1235.

[2] H. Blasius, "Laminare Stromung in Kanalen Wechselnder", Breite, Z. Math. Phys. 58 (1910) 225-233.

[3] J. F. Brady and A. Acrivos, "Steady flow in a channel or tube with an accelerating surface velocity. An exact solution to the Navier-Stokes equations with reverse flow", J. Fluid Mech. 112 (1981) 127-150.

[4] C. Domb and M. F. Sykes, "On the susceptibility of a ferromagnetic above the curie point", Proc. R. Soc. London, Ser. A 240 (1957) 214-228.

[5] P. G. Drazin and Y. Tourigny, "Numerical study of bifurcation by analytic continuation of a function defined by a power series", SIAM J. Appl. Math. 56 (1996) 1-18.

[6] M. Van Dyke, "Analysis and improvement of perturbation series", Quart. J. Mech. Appl. Math. 27 (1974) 423-449.

[7] L. E. Fraenkel, "Laminar flow in symmetrical channels with slightly curved walls. I: On the Jeffery-Hamel solutions for flow between plane walls", Proc. R. Soc. Lond. Ser. A 267 (1962) 119-138.

[8] D. S. Gaunt and A. J. Gutmann, Phase transitions and critical phenomena, 3 ed. (1974).

[9] R. D. Lucas, "A perturbation solution for viscous incompressible flow in channels", Ph. D. Thesis, Stanford University, 1972.

[10] J. N. Lyness, "Differentiation formulas for analytic functions", Math. Comput. 21 (1967) 352-362.

[11] O. D. Makinde, "Unsteady incompressible flow in a porous channel", Proc. Appl. Indust. Maths. Conf. Rom. Oradea (1994) 47-58.

[12] O. D. Makinde, "Laminar flow in a channel of varying width with permeable boundaries", Rom. J. Phys. 40 (1995) 403-417.

[13] O. D. Makinde, "Computer extension and bifurcation study by analytic continuation of the porous tube flow problem", J. Math. Phys, Sci. 30 (1996) 1-24.

[14] O. D. Makinde, "Steady flow in a linearly diverging asymmetrical channel", CAMES 4 (1997), 157-165.

[15] O. D. Makinde and E. M. Lungu, "On the flow of a viscous fluid driven along a tube by a moving surface", J. Inst. Math. Comp. Sci. 8 (1997) 87-98. 
[16] T. J. Pedley, The Fluid Mechanics of Large Blood Vessels (Cambridge University Press, Cambridge, 1980).

[17] R. M. Terrill and P. W. Thomas, "Laminar flow through a uniformly porous pipe", Appl. Sci. Res. 21 (1969) 37-67.

[18] M. M. Vainberg and V. A. Trenogin, Theory of branching of solutions of nonlinear equations (Noordoff, Leyden, 1974).

[19] M. B. Zaturska and W. H. H. Banks, "Flow in a pipe driven by suction at an accelerating wall", Acta Mechanica 110 (1995) 111-121. 